
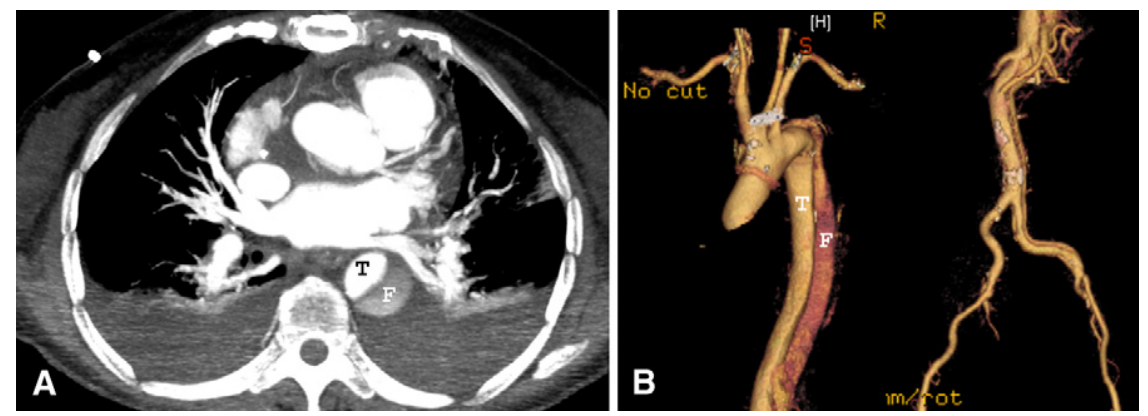

FIGURE 3. Axial (A) and 3-dimensional (B) postoperative CT angiography showing the dissection extending down the aorta to the level of iliac vessels and true and false lumens. $F$, False; $T$, true.

perfusion might be maintained as the flap returns back to the aorta in systole. This was most likely the case in our patient because there was "late ghosting" of the left coronary system and no decrease in left ventricular function despite the appearance of total occlusion of the LMCA, and coronary artery bypass graft was not warranted.

\section{CONCLUSIONS}

In cases of acute aortic dissection, the potential for a circumferential intimal tear needs to be considered because intimo-intimal intussusception, although rare, is a serious complication of aortic dissection. Coronary angiography may indicate complete coronary artery occlusion that may be due to the intussuscepting flap and will resolve once the aortic defect has been repaired. Intraoperative
TEE is the best investigative modality before definitive management with operative repair.

\section{References}

1. Yavuz S, Elhan K, Eris C, Tugrul Goncu M. Intimo-intimal intussusception: a rare clinical form of aortic dissection. Eur J Cardiothorac Surg. 2003;23:850-1; author reply 851

2. Chow JL, Mariano ER, Liang D. Transesophageal echocardiography assessment of severe aortic regurgitation in type a aortic dissection caused by a prolapsed circumferential intimal flap. J Cardiothorac Vasc Anesth. 2007;21:85-7.

3. Yamabi H, Imanaka K, Sato H, Matsuoka T. Extremely localized aortic dissection and intussusception of the intimal flap into the left ventricle. Ann Thorac Cardiovasc Surg. 2011;17:431-3.

4. Yavuz S, Eris C, Turk T. Re: Severe aortic regurgitation caused by a prolapsed circumferential intimal flap in acute type A aortic dissection: role of intraoperative transesophageal echocardiography? J Cardiothorac Vasc Anesth. 2008;22:941.

5. Massetti M, Neri E, Babatasi G, et al. Flap suffocation: an uncommon mechanism of coronary malperfusion in acute type A dissection. J Thorac Cardiovasc Surg. 2003;125:1548-50.

\title{
Pulsatile biventricular assist device and an absent mitral valve
}

\author{
Igor Gosev, MD, Stephanie L. Mick, MD, Gregory S. Couper, MD, and Prem S. Shekar, MD, Boston, Mass
}

We report the case of a 23-year-old African American woman with a history of discoid lupus erythematosus who presented with severe mitral valve insufficiency and preserved left ventricular function. The initial attempt at repair was unsuccessful, and she received a bioprosthetic valve replacement. Sequential valve dehiscence resulted in 2 subsequent reoperations and repeat valve replacements with

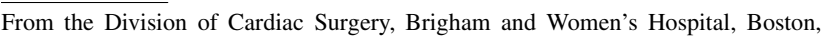
Mass.

Disclosures: Authors have nothing to disclose with regard to commercial support. Received for publication April 9, 2012; accepted for publication May 7, 2012.

Address for reprints: Igor Gosev, MD, Division of Cardiac Surgery, Brigham and Women's Hospital, 15 Francis St, 02115, Boston, MA (E-mail: igosev@ partners.org).

J Thorac Cardiovasc Surg 2012;144:e23-5

$0022-5223 / \$ 36.00$

Copyright (c) 2012 by The American Association for Thoracic Surgery

http://dx.doi.org/10.1016/j.jtcvs.2012.05.011
}

a bioprosthesis and mechanical prosthesis, respectively. She underwent testing for endocarditis that was found to be negative on both occasions, but she did receive empiric endocarditis therapy on both occasions.

Two weeks after the third operation, florid biventricular failure from a recurrent valve dehiscence developed in the patient. There was no impairment of the mechanical leaflet motion. Emergency surgery was performed.

At surgery, it was found that the mitral valve prosthesis had completely dehisced from a nearly nonexistent annulus. Mitral valve replacement was deemed impossible, so the mechanical valve was explanted and a pulsatile biventricular assist device (BIVAD, Thoratec Corporation, Pleasanton, Calif) was placed for biventricular dysfunction.

The patient's early postoperative period was complicated by hypoxic respiratory failure and inability to be weaned from respiratory support. Chest x-rays showed florid 


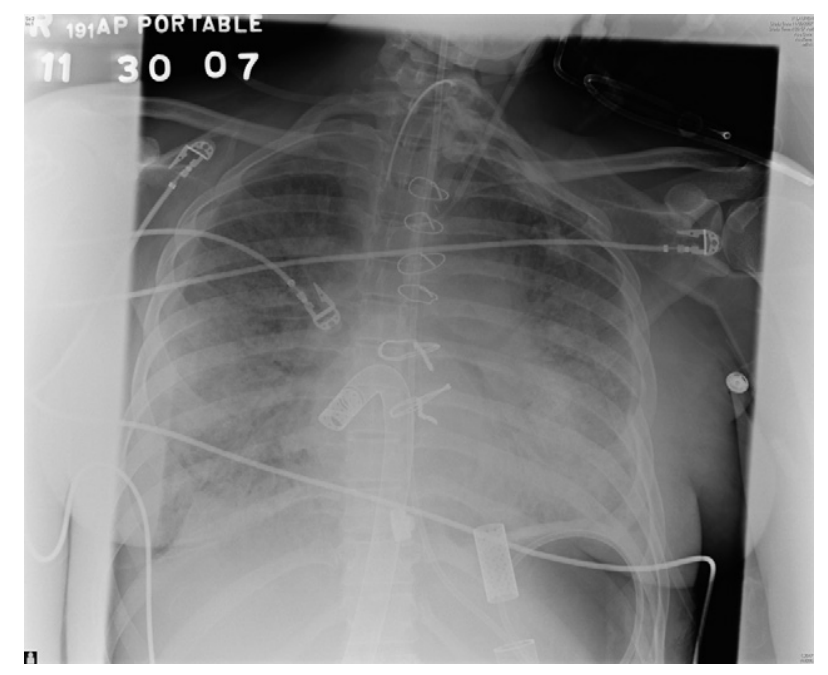

FIGURE 1. Chest x-ray showing severe pulmonary edema.

pulmonary edema (Figure 1). The BIVAD function was normal. Transesophageal echocardiography revealed torrential regurgitation through a wide-open mitral annulus, flooding her lungs (Figure 2).

Despite the presence of the properly functioning BIVAD, blood was propelled backward by a left ventricle that had since recovered and was discordant with the ventricular assist device rhythm. Re-replacement of the mitral valve with explantation of the BIVAD was considered a nonviable option given the sequential dehiscence of 3 prostheses with no discernable cause and the lack of available annular tissue for a fourth operation. Her only available option was orthotopic heart transplantation provided her condition could be stabilized.

It seemed physiologically appropriate to reduce her native ventricular rate to minimize the retrograde propulsion of blood into the lungs with every native beat. Maximal doses of multiple nodal agents did not achieve the desired physiologic result. An atrioventricular junction ablation was performed, and despite a heart rate of 25 beats/min, the florid pulmonary edema continued unabated. Transesophageal echocardiography continued to show marked reversal of flow within the pulmonary veins despite a low ventricular rate and now moderately reduced left ventricular function. Her ventilator dependence continued.

In an effort to resolve the pulmonary edema by optimal left ventricular unloading and minimizing the retrograde propulsion, the patient's left ventricular assist device (LVAD) was changed to a continuous-flow assist device (Levitronix CentriMag; Thoratec Corporation). This was done without sternal reentry or cardiopulmonary bypass using the existing inflow and outflow cannula of the pulsatile LVAD. The pulsatile right ventricular assist device was unchanged.

With the continuous-flow LVAD, her pulmonary edema improved and eventually resolved. On the tenth

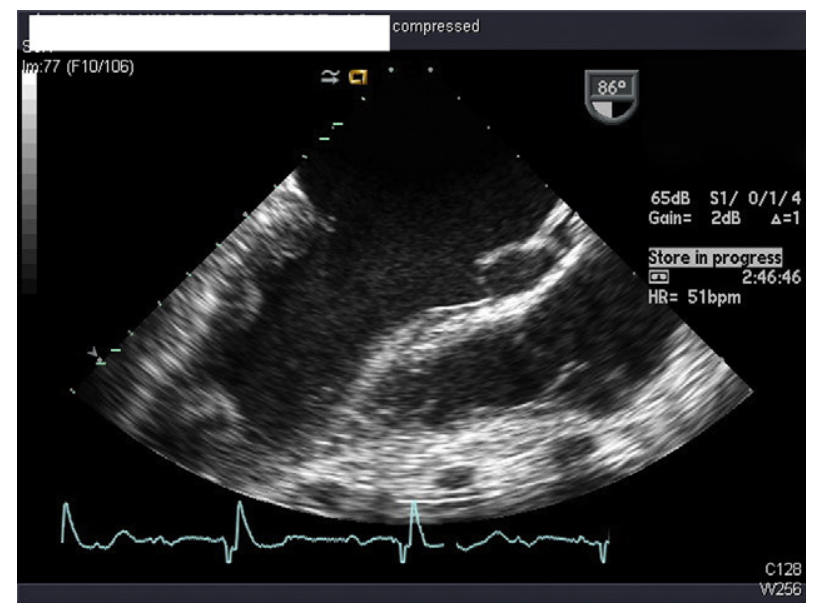

FIGURE 2. Echocardiography showing left ventricle without mitral valve and with ventricular assist device.

postoperative day, transesophageal echocardiography showed that the retrograde flow was now largely absent, and the left side of the heart was fully unloaded. She was gradually weaned from the ventilator and placed on the heart transplant list.

After 105 days of left ventricular support with the continuous-flow LVAD and a pulsatile right ventricular assist device, the patient underwent successful orthotopic heart transplantation. She has made a complete recovery and is currently completely functional.

\section{DISCUSSION}

The tissue quality of patients with antiphospholipid antibody syndrome is often poor with signs of chronic inflammation and fibrinoid and myxoid degenerative transformation. ${ }^{1,2}$ Severe degenerative changes in the mitral annulus of our patient likely caused recurrent destabilization of the mitral prosthesis. Although her presentation at the third valve dehiscence was one of severe biventricular dysfunction requiring BIVAD, her native function quickly recovered. We believe that the subsequent inability to synchronize native contraction of the preserved left ventricle with pulsatility of the LVAD in the setting of an absent mitral valve led to "flooding" of the pulmonary veins from a large regurgitant volume. With the change to a nonpulsatile device, continuous unloading of the left ventricle became possible, and this minimized regurgitant volume to the left atrium and lungs. This led to the resolution of the pulmonary edema, allowing us to wean her from the respirator.

\section{CONCLUSIONS}

The centrifugal continuous-flow LVAD showed excellent midterm durability and superior unloading of the left ventricle in the absence of the mitral valve and 
preserved left ventricular function, when compared with the pulsatile device. The cascade of unique physiologic events that we confronted in the management of our patient was a singular learning event that leads to this case report.

\section{References}

1. Carvera R, Font J, Pare C, et al. Cardiac disease in systemic lupus erythematosus: prospective study of 70 patients. Ann Rheum Dis. 1992;51:156-9.

2. Bouma W, Klinkenberg TJ, Van der Horst I, et al. Mitral valve surgery for mitra regurgitation caused by Lieman-Sacks endocarditis: a report of four cases and a systematic review of the literature. J Cardiothorac Surg. 2010;5:13.

\title{
Management of an aortoesophageal fistula caused by Kirschner wire migration in a patient with arteria lusoria
}

\author{
Bastien Orsini, MD, ${ }^{\mathrm{a}}$ Philippe Amabile, MD ${ }^{\mathrm{b}}$ Laurence Bal, MD ${ }^{\mathrm{b}}$ and Philippe Piquet, MD,${ }^{\mathrm{b}}$ Marseille, \\ France
}

Intrathoracic wire migration after orthopedic surgery is a rare complication that most often happens after the treatment of clavicular fractures and dislocation of the sternoclavicular and acromioclavicular joints. We describe here, for the first time to our knowledge, an aortoesophageal fistula caused by intrathoracic Kirschner wire migration.

\section{CLINICAL SUMMARY}

A 58-year-old man treated 6 years previously with a Kirschner wire for a fractured left clavicle was admitted to the hospital for acute thoracic pain. Radiographic examination revealed migration of the wire from the left clavicle to the mediastinum, through the ostium of the left subclavian artery, which was thrombosed, just above the aortic arch. There was no hemomediastinum or pneumomediastinum. We observed that the patient had an arteria lusoria (Figure 1). The tip of the wire was located in contact with the esophagus with an infiltration of mediastinal fat. The patient was in hemodynamically stable condition. Esophageal and bronchial endoscopies were planned, but the patient, who was deaf and nonverbal with mental retardation, refused these examinations.

Open repair was attempted through a median sternotomy. Wire extraction was not possible with this incision alone. A left supraclavicular cervical incision and an anterior thoracotomy in the first left intercostal space were then necessary to control the left subclavian artery and to find the

\footnotetext{
From the Thoracic Surgery Department, ${ }^{\text {a }}$ Hôpital Nord, Marseille, France; and the Vascular Surgery Department, ${ }^{\text {b }}$ Hôpital de la Timone, Marseille, France. Disclosures: Authors have nothing to disclose with regard to commercial support. Received for publication Feb 2, 2012; revisions received April 10, 2012; accepted for publication May 9, 2012; available ahead of print June 7, 2012.

Address for reprints: Philippe Amabile, MD, Aix-Marseille Univ, 13284 Marseille, France and APHM, Department of Vascular Surgery, Hôpital de la Timone, 13385 Marseille, France (E-mail: philippe.amabile@ap-hm.fr)

J Thorac Cardiovasc Surg 2012;144:e25-7

$0022-5223 / \$ 36.00$

Copyright (c) 2012 by The American Association for Thoracic Surgery

http://dx.doi.org/10.1016/j.jtcvs.2012.05.022
}

proximal end of the wire. During extraction, there was no bleeding observed in the surgical site, but blood quickly appeared in the gastric tube, followed by hemorrhagic shock from an aortoesophageal fistula. It was not possible to repair this fistula by sternotomy. A Sengstaken-Blakemore tube was inflated in the esophagus to stop bleeding. The patient was transferred to the intensive care unit for hemodynamic stabilization and then transferred 24 hours later to our hospital, where he was immediately treated.

We decided that an endovascular procedure would be the most appropriate, because there were anticipated difficulties in the surgical control of the posterior aortic arch. The right subclavian artery was transposed in the right common carotid artery, and an aortic stent graft (Cook Inc, Bloomington, Ind) was successfully implanted. Because of the lack of symptoms of left subclavian occlusion, revascularization was not performed. No esophageal injury was seen in the endoscope, so no other complementary procedure was conducted. Broad-spectrum antibiotic treatment was prescribed for 6 weeks.

During postoperative recovery, the patient had difficulties with respiratory weaning that required a tracheotomy and a left pleural decortication. Oral feeding was started without difficulty. Computed tomographic scan showed permeability of the transposition, thrombosis of the lusoria (Figure 2), and absence of mediastinitis. After 1 year of follow-up, the patient is doing well and has regained his preoperative physical state.

\section{DISCUSSION}

Intrathoracic wire migration after orthopedic surgery is a rare complication but has regularly been reported since the first description written by Mazet. ${ }^{1}$ This most often happens after the treatment of clavicular fractures and dislocation of the sternoclavicular and acromioclavicular joints. Many articles have examine the state of tracheal, cardiac, vascular, and esophageal injuries, ${ }^{2-4}$ but to our knowledge 